\title{
Fluorometric Study on the Interaction between Lomefloxacin and Bovine Lactoferrin
}

\author{
Xiang Chen, Ji-Cai Fan, Yun Wang, Cheng-Ping Fan, and Zhi-Cai Shang ${ }^{\dagger}$ \\ Department of Chemistry, Zhejiang University, Hangzhou 310027, China
}

\begin{abstract}
The binding of lomefloxacin to bovine lactoferrin (BLf) in a dilute aqueous solution was studied using fluorescence spectra. The binding constant $(K)$ and the number of binding sites $(n)$ were obtained by a fluorescence quenching method. The binding distance $(r)$ and energy-transfer efficiency $(E)$ between lomefloxacin and bovine lactoferrin were also obtained according to the mechanism of Foörster-type dipole-dipole nonradiative energy-transfer. The effect of lomefloxacin on the conformation of bovine lactoferrin was also analyzed by synchronous fluorescence spectroscopy. The interaction between lomefloxacin and bovine lactoferrin is strong. Lomefloxacin can affect the conformation of bovine lactoferrin to some degree.
\end{abstract}

(Received April 7, 2005; Accepted September 5, 2005)

\section{Introduction}

Lactoferrin is an $80 \mathrm{kDa}$ iron-binding glycoprotein that is present in several biological fluids and in the secretory granules of neutrophils. ${ }^{1-3}$ It has been associated with a wide variety of biologically important processes, including host defense, regulation of cell growth, and cell differentiation. ${ }^{4}$ Lactoferrin is folded into two approximately equal lobes ( $\mathrm{N}$ - and $\mathrm{C}$ terminal), each of which is split by an iron-binding cleft into two domains (N1, N2 and $\mathrm{C} 1, \mathrm{C} 2$ respectively). ${ }^{5}$ It is a highly basic protein, and consequently interacts with many acidic molecules. ${ }^{6}$ Such interactions may modify the biological properties of lactoferrin. This interaction between protein and drug molecules results in a stable protein-drug complex being formed. Studying binding phenomena will be important for interpretating the metabolism and transporting process, and will help to explain the relationship between the structures and functions of proteins.

In recent years, interactions between drugs of quinolones and protein molecules ${ }^{7,8}$ have been widely studied. In the interest of enriching the information database of such research, we chose lomefloxacin (LMF, Fig. 1) as a donor, which is a typical representation of quinolones. Lomefloxacin is an antibiotic in a class of drugs called fluoroquinolones with a wide spectrum of activity against various bacterial infections, such as bronchitis and urinary tract infections. ${ }^{9}$ There have been lots of reports on the interaction between lomefloxacin and serum albumiln. ${ }^{8}$ However, because few reports on the interaction between lomefloxacin and bovine lactoferrin (BLf) can be obtained, we became interested in studying the binding reaction in such a solution system.

Fluorescence spectroscopy is an appropriate method to determine the interaction between a small-molecule ligand and a bio-macromolecule. From measurements and analyses of the emission peak, the transfer efficiency of energy, the lifetime,

$\doteqdot$ To whom correspondence should be addressed.

E-mail: shangzc@zju.edu.cn and fluorescence polarization etc., a vast amount of information will be obtained about the structural fluctuations and the microenvironment of the fluorophore in macromolecules.

In the present work, the binding reaction between lomefloxacin and BLf was investigated; also, the binding parameters and transfer efficiency of energy were measured. Another main purpose of this work was to check the effect of lomefloxacin on BLf conformational changes. Such conformational changes can be evaluated by measuring the synchronous fluorescence ${ }^{10-15}$ intensity. Based on the sitebinding model, ${ }^{16-19}$ practical fitting formulas for drugs binding to BLf were proposed. The results show that these models can be responsible for experiments.

\section{Experimental}

\section{Apparatus and reagents}

All of the fluorescence measurements were carried out on a F4500 recording spectrofluorometer (Hitachi, Japan) equipped with a xenon lamp source and $1.0 \mathrm{~cm}$ cells. A SPECORD 200 recording spectrophotometer (Analytik Jena AG, Germany) equipped with a $1.0 \mathrm{~cm}$ cuvette was used to scan the UV spectrum. All pH measurements were made with a PHS-3C digital pH-meter (Yitong Company of Jintan, Jiangsu China). BLf (Sigmag) and lomefloxacin (Xinchang Pharmacy Factory,<smiles>CCCn1cc(C(=O)O)c(=O)c2cc(F)c(N3CCNC(C)C3)c(F)c21</smiles>

Fig. 1 Chemical structure of lomefloxacin. 


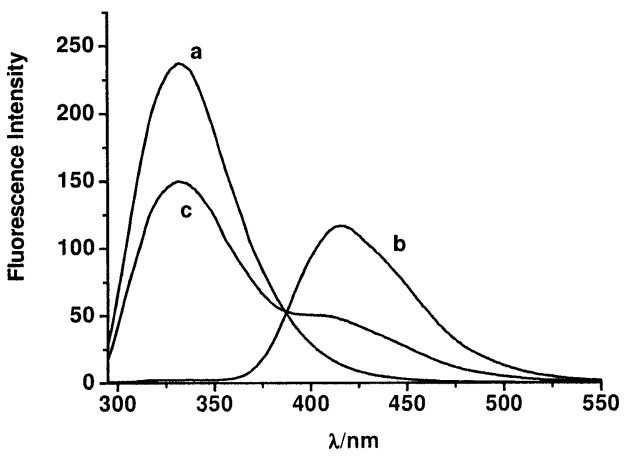

Fig. 2 Fluorescence spectra of lomefloxacin (LMF) and BLf (pH, 7.0; $\lambda_{\mathrm{ex}}, 290 \mathrm{~nm}$; a, BLf; b, LMF; c, BLf + LMF (1:1)).

\section{Zhejiang China) were used.}

Solutions of BLf $\left(1.00 \times 10^{-5} \mathrm{~mol} \mathrm{~L}{ }^{-1}\right)$, lomefloxacin $(5.0 \times$ $\left.10^{-4} \mathrm{~mol} \mathrm{~L}^{-1}\right)$ and $0.05 \mathrm{~mol} \mathrm{~L}^{-1}$ Tris- $\mathrm{HCl}$ buffer of $\mathrm{pH}=7.0(0.1$ mol L-1 $\mathrm{NaCl}$ was used to keep the ionic strength constant) were prepared. All reagents were of analytical grade, and doubledistilled water was used throughout.

\section{Procedures}

BLf and lomefloxacin were dissolved in Tris- $\mathrm{HCl}$ buffer; the concentrations of BLf and lomefloxacin were $1.00 \times 10^{-5} \mathrm{~mol}$ $\mathrm{L}^{-1}$ and $5.0 \times 10^{-4} \mathrm{~mol} \mathrm{~L}^{-1}$, respectively. To a $1.0 \mathrm{~cm}$ quartz cell, the BLf solution was added to $2.5 \mathrm{ml}$, and the range of the drug solution was gradually added into the cell using a microinjector. The accumulated volumes were 20, 40, 60, 80, 100, $120,140,160$, and $180 \mu \mathrm{l}$ (total accumulated volume $<200 \mu \mathrm{l}$ ). Under the apparatus condition of both the entrance slit and the exit slit width being $5 \mathrm{~nm}$, and the scanning speed being $240 \mathrm{~nm}$ $\mathrm{min}^{-1}$, fluorescence quenching spectra and synchronous fluorescence spectra were obtained. Fluorescence quenching spectra were obtained at excitation and emission wavelengths of $295 \mathrm{~nm}$ and $300-550 \mathrm{~nm}$, respectively. The range of synchronous scanning was $270-315 \mathrm{~nm}$ and $235-305 \mathrm{~nm}$, where the differences in the wavelengths $(\Delta \lambda)$ were $60 \mathrm{~nm}$ and $15 \mathrm{~nm}$ separately. For absorption spectra (UV) experiments, samples of lomefloxacin were brought to $1.0 \mathrm{~cm}$ cuvettes versus a blank of buffer. The absorbances were read and a spectral scanning curve was made.

\section{Results and Discussion}

Fluorescence spectra

Figure 2 shows the fluorescence spectra of lomefloxacin and BLf. The fluorescence emission lines of the lomefloxacin-BLf (1:1) mixture system can also be observed in Fig. 2. It is wellknown that BLf has strong fluorescence at $\lambda_{\mathrm{ex}} / \lambda_{\mathrm{em}}=290 / 332$ $\mathrm{nm}$. When the solution condition agrees with our studies, Fig. 2 shows the maximum strong fluorescence at $415 \mathrm{~nm}$ for lomefloxacin. In addition, the BLf fluorescence spectra is somewhat quenched, which means that interaction has occured and the energy has been transferred.

\section{Binding constant and binding sites}

For experiments carried out at large molar protein/dye ratios, it was assumed that only strong sites were active in the binding dye. For simplicity, these strong binding sites were also assumed to be identical, and to act independently. If these

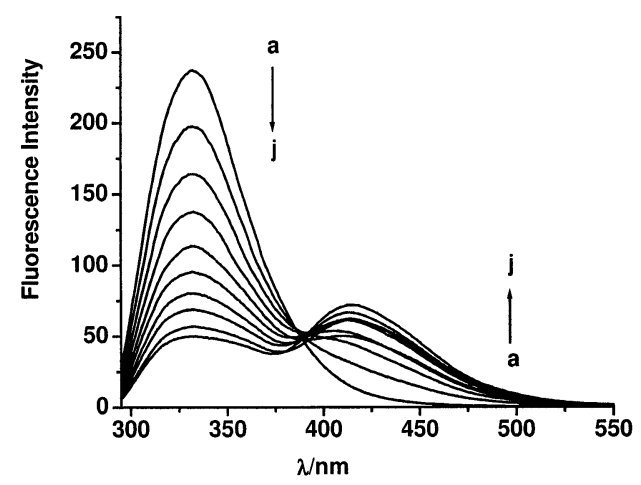

Fig. 3 Effect of the lomefloxacin concentration on the quenching of BLf fluorescence $\left(\mathrm{pH}, 7.0 ; \lambda_{\mathrm{ex}}, 290 \mathrm{~nm}\right.$; [BLf], $1 \times 10^{-5} \mathrm{~mol} \mathrm{~L}^{-1}$; [LMF], $10^{-5} \mathrm{~mol} \mathrm{~L}^{-1}$; from a to $\mathrm{j}: 0,0.4,0.8,1.2,1.6,2.0,2.4,2.8$, $3.2,3.6)$.

assumptions are valid, a site-binding model could be constructed, and a binding equation described by Scatchard is given by ${ }^{17,18}$

$$
v=n K[\mathrm{D}] /(1+K[\mathrm{D}])
$$

Here, $v$ is the average number of dye molecules bound per protein molecule, $n$ is the number of (strong) binding sites, $K$ is the intrinsic (microscopic) binding (association) constant, and [D] is the concentration of free (unbound) dye.

A linear form of the equation is

$$
v /[\mathrm{D}]=-K v+K n \text {. }
$$

Equation (2) is the usual form of the Scatchard equation. ${ }^{17,18}$

In the case of fluorescence only caused by a protein at a selected wavelength, the relationship between the concentration of protein and the fluorescence intensity can be described by

$$
F_{0} / F=\left[\mathrm{P}_{\mathrm{t}}\right] /[\mathrm{P}] .
$$

According to the definition of $n$, another equation is also known,

$$
v=\left(\left[\mathrm{D}_{\mathrm{t}}\right]-[\mathrm{D}]\right) /\left[\mathrm{P}_{\mathrm{t}}\right]=n\left(\left[\mathrm{P}_{\mathrm{t}}\right]-[\mathrm{P}]\right) /\left[\mathrm{P}_{\mathrm{t}}\right]=n\left(F_{0}-F\right) / F_{0},
$$

where, $\left[\mathrm{P}_{\mathrm{t}}\right]$ is the total protein concentration, and $\left[\mathrm{D}_{\mathrm{t}}\right]$ is the final dye concentration; $F_{0}$ and $F$ are, respectively, the fluorescence intensity in the absence of a quencher and in its presence at [D] concentration.

The following equation is obtained by combining Eqs. (1) and (4):

$$
F_{0} / F=K\left[\mathrm{D}_{\mathrm{t}}\right] F_{0} /\left(F_{0}-F\right)-n K\left[\mathrm{P}_{\mathrm{t}}\right]
$$

It indicates that the binding constant $(K)$ and binding sites $(n)$ can be obtained at the same time using a least-squares algorithm for data-fitting according to Eq. (5).

The fluorescence quenching spectra of BLf in Tris- $\mathrm{HCl}$ buffer with increasing lomefloxacin concentration and fixed BLf concentration $\left(1.00 \times 10^{-5} \mathrm{~mol} \mathrm{~L}^{-1}\right)$ are shown in Fig. 3. It is obvious that the concentration of lomefloxacin gradually increases with the addition, the fluorescence intensity of BLf decreases regularly at the same time, and the fluorescence emission peak of lomefloxacin is gradually enhanced. A well- 
Table 1 Binding parameters for the system of lomefloxacin-BLf

\begin{tabular}{ccc} 
Binding constant & Binding site & Correlation coefficient \\
$K=1.64 \times 10^{5} \mathrm{~L} \mathrm{~mol}^{-1}$ & $n=1.76$ & $\gamma=0.995$ \\
\hline
\end{tabular}

defined isosbestic point is observed at $390 \mathrm{~nm}$, which is direct evidence of drug-protein complex formation.

After the fluorescence quenching on BLf at $332 \mathrm{~nm}$ was measured, the linear fit of the fluorescence intensity changes of the BLf-LMF system was assessed by Eq. (5). Figure 4 shows the fitting curve and Table 1 shows the fitting results.

It is indicated that there is a strong binding force between lomefloxacin and BLf, and that about two binding sites would be formed. Here, we found that the number of the binding sites was the same as that for iron, but it is uncertain that the binding sites for lomefloxacin are equal to those for iron. Maybe because of the symmetry of the lactoferrin molecule, two binding sites were formed. The two binding sites should have the same binding constant according to Scatchard equation. A Scatchard plot obtained in the present work was one straight line, which can validate the rationality for the binding model. The property of symmetry may be a contributing factor. The two binding sites have similar structural environments due to such molecular symmetry.

Binding distance between the drug and the amino acid residues of $B L f$

According to the Förster non-radioactive energy transfer theory, ${ }^{20}$ the energy-transfer effect is related not only to the distance between the acceptor and donor $(r)$, but also to the critical energy transfer distance $\left(R_{0}\right)$.

$$
E=R_{0}{ }^{6} /\left(R_{0}{ }^{6}+r^{6}\right),
$$

where $R_{0}$ is the critical distance when the transfer efficiency is $50 \%$,

$$
R_{0}{ }^{6}=8.8 \times 10^{-25} \mathrm{~K}^{2} \mathrm{~N}^{-4} \Phi J .
$$

Here, $K^{2}$ is the spatial-orientation factor of the dipole, $N$ is the refractive index of the medium, $\Phi$ is the fluorescence quantum yield of the donor, and $J$ is the overlap integral of the fluorescence emission spectrum of the donor and the absorption spectrum of the acceptor. ${ }^{21,22}$ Therefore,

$$
J=\int_{0}^{\infty} F(\lambda) \varepsilon(\lambda) \lambda^{4} \mathrm{~d} \lambda / \int_{0}^{\infty} F(\lambda) \mathrm{d} \lambda .
$$

Here, $F(\lambda)$ is the fluorescence intensity of the fluorescent donor at wavelength $\lambda$, and $\varepsilon(\lambda)$ is the molar absorptivity of the acceptor at wavelength $\lambda$. Thus the energy transfer efficiency is

$$
E=1-F / F_{0}
$$

The overlap of the absorption spectrum of lomefloxacin and the fluorescence emission spectrum of BLf is shown in Fig. 5. The overlap integral $(J)$ can be evaluated by integrating the spectra in Fig. 5. In this paper, $J$ is given by following equation (calculated to be $1.33 \times 10^{-14} \mathrm{~cm}^{3} \mathrm{dm}^{3} \mathrm{~mol}^{-1}$ ):

$$
J=\sum F(\lambda) \varepsilon(\lambda) \lambda^{4} \Delta \lambda / \sum F(\lambda) \Delta \lambda
$$

Under these experimental conditions, the distance corresponding to $50 \%$ energy transfer from BLf to lomefloxacin

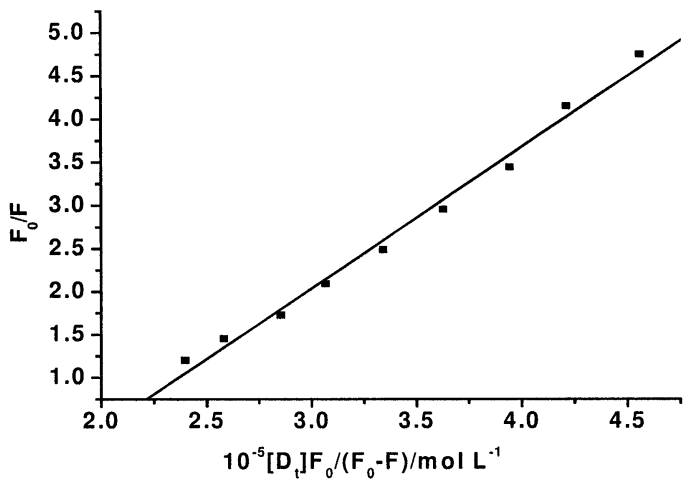

Fig. 4 Fitting curve of the lomefloxacin-BLf solution system (pH, $7.0 ;$ [BLf], $1 \times 10^{-5} \mathrm{~mol} \mathrm{~L}^{-1} ; \lambda_{\mathrm{ex}}, 290 \mathrm{~nm} ; \lambda_{\mathrm{em}}, 332 \mathrm{~nm}$ ).

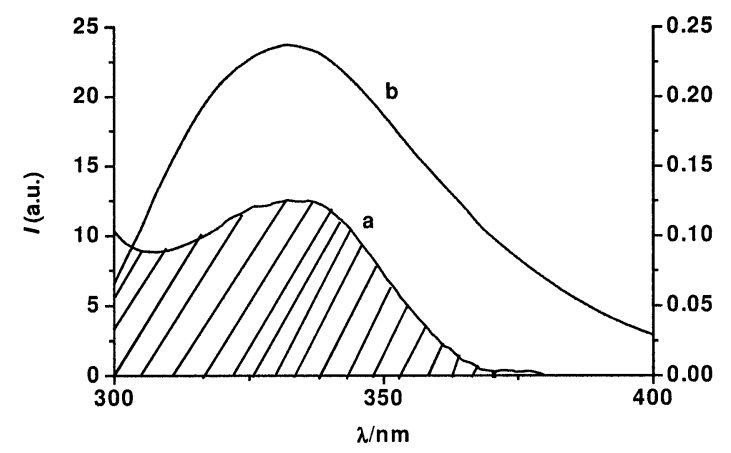

Fig. 5 Overlap of the absorption spectrum of lomefloxacin (a) with the fluorescence emission spectrum of BLf (b).

can be estimated to be $R_{0}=2.58 \mathrm{~nm}$ from Eq. (7) using $K^{2}=2 / 3$, $N=1.336, \Phi=0.118 .^{23-25}$ Moreover, the energy transfer effect is $E=0.369$ from Eq. (6), and the binding distance between lomefloxacin and amino acid residue in BLf is $r=2.82 \mathrm{~nm}$.

\section{Effect of the drug on the BLf conformation}

The conformational changes of BLf were evaluated by measuring the synchronous fluorescence intensity of protein amino acid residues before and after the addition of lomefloxacin. Fluorescence measurements provide information about the molecular environment in the vicinity of the fluorophore functional groups. In the synchronous spectra, the sensitivity associated with fluorescence is maintained while offering several advantages: spectral simplification, spectral bandwidth reduction and avoiding different perturbing effects. In the present work, synchronous fluorescence spectroscopy was used to study the synchronous fluorescence characteristics of BLf at different scanning intervals, $\Delta \lambda\left(\Delta \lambda=\lambda_{\text {emission }}-\right.$ $\left.\lambda_{\text {excitation}}\right)$. When $\Delta \lambda=15 \mathrm{~nm}$, the spectrum characteristic of the protein tyrosine residues was observed, and when $\Delta \lambda=60 \mathrm{~nm}$, the spectrum characteristic of protein tryptophan residues was observed. The authors suggested ${ }^{26,27}$ that a useful method to study the environment of amino acid residues is to measure a possible shift in the wavelength emission maximum $\left(\lambda_{\max }\right)$. The shift in the position of the emission maximum corresponds to changes in the polarity around the chromophore molecule. Thus, the conformation changes of BLf can be evaluated by measuring $\lambda_{\max }$.

With the concentration of protein being unchanged, and the concentration of lomefloxacin increasing by addition, the synchronous spectroscopy was scanned at $\Delta \lambda=15 \mathrm{~nm}$, and 

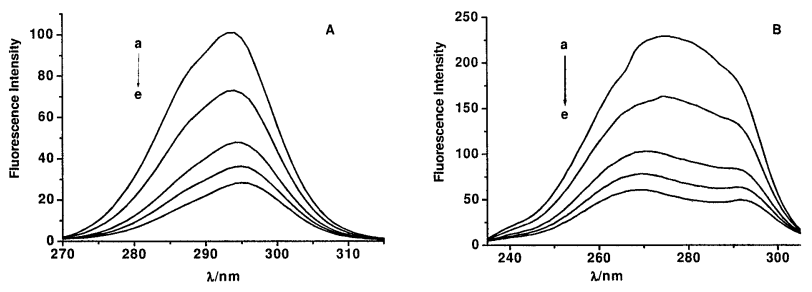

Fig. 6 Effect of drug on the synchronous fluorescence spectra of BLf (A, $\Delta \lambda=15 \mathrm{~nm}$; B, $\Delta \lambda=60 \mathrm{~nm}$; [BLf], $1 \times 10^{-5} \mathrm{~mol} \mathrm{~L}^{-1}$; [Lomefloxacin], $10^{-5} \mathrm{~mol} \mathrm{~L}^{-1}$; from a to e: $0,0.8,1.6,2.4,3.4$ ).

$\Delta \lambda=60 \mathrm{~nm}$ (shown in Fig. 6).

The effect of lomefloxacin on the tyrosine and tryptophan residues fluorescence intensity of BLf indicates that the main contribution to the fluorescence intensity of BLf is tryptophan residues. Figure 6 also shows the effect of lomefloxacin on the emission maximum with the procession of titration. A slightly stronger blue-shift of tryptophan fluorescence upon the addition of drug was observed, and the emission maximum of tyrosine kept its position. This shift indicates that tryptophan residues were placed in a more hydrophobic environment and less exposed to the solvent. This may be due to the lomefloxacin insert in BLf molecules, and a consequent rearrangement of the tryptophan microenvironment. It is also suggested that the environments of tryptophan residues in pure protein solution are relatively polar. Binding of the lomefloxacin changes the environments to apolar ones. A shift in polarity is brought about by conformational changes derived from the interaction between protein and the ligand molecule.

\section{References}

1. P. L. Masson, J. F. Heremans, and J. H. Dive, Clin. Chim. Acta, 1966, 14, 735.

2. P. L. Masson, J. F. Heremans, and E. Schonne, J. Exp. Med., 1969, 130, 643.

3. R. M. Bennett, A. C. Eddie-Quartey, and P. J. L. Holt, Arthrit. Rheumat, 1973, 16, 186.

4. L. Sanchez, M. Calvo, and J. H. Brock, Arch. Dis. Child, 1992, 7, 657 .
5. E. N. Baker, B. F. Anderson, H. M. Baker, R. T. A MacGillivray, S. A. Moore, N. A. Peterson, S. C. Shewry, and J. W. Tweedie, Adv. Exp. Med. Biol., 1998, 43, 1.

6. F. Lampreave, A. Pineiro, J. H. Brock, H. Castillo, L. Sanchez, and M. Calvo, Int. J. Biol. Macromol., 1990, 12, 2.

7. Y. Xu and H. X. Shen, Chin. J. Anal. Chem., 1998, 26, 1494.

8. J. He, S. Q. Wu, F. X. Li, X. G. Chen, and Z. D. Hu, Chem. J. Lanzhou University, 2004, 40, 54.

9. J. M. Blondeau, Clin. Therapeutics, 1999, 21, 3.

10. J. B. F. Lloyd, Nature Phys. Sci., 1971, 231, 64.

11. J. B. F. Lloyd, Nature, 1974, 99, 729.

12. J. B. F. Lloyd, Nature, 1975, 100, 82.

13. Q. Q. Xu, L. M. Du, and J. P. Wang, Spectropsc. Spectral Anal., 2002, 22, 444.

14. E. L. Inman and J. D. Winefordner, Anal. Chim. Acta, 1982, 138, 245.

15. E. L. Inman and J. D. Winefordner, Anal. Chem., 1982, 54, 2018.

16. I. M. Klotz and D. L. Hunstone, Biochemistry, 1971, 10, 3065.

17. R. W. Congdon, G. W. Muth, and A. G. Splittgerber, Anal. Biochem., 1993, 213, 407.

18. S. B. Mauricio and L. I. Guiherme, J. Phys. Chem., 1998, 102, 4678.

19. G. Scatchard, Ann. NY Acad. Sci., 1949, 51, 660.

20. D. William, J. Horrocks, and E. C. William, J. Am. Chem. Soc., 1981, 103, 2826.

21. C. Q. Jiang, M. X. Gao, and J. X. He, Anal. Chim. Acta, $\mathbf{2 0 0 2}, 452,185$.

22. N. Shaklai, J. Yguerabide, and H. M. Ranney, Biochemistry, 1977, 16, 5585.

23. J. M. Vanderkooi, A. Ierokomas, H. Nakmura, and A. Martonosi, Biochemistry, 1977, 16, 1262.

24. L. Cyril, J. K. Earl, and W. M. Sperry, "Biochemists Handbook", 1961, E \& EN Spon, London.

25. B. L. Zhang, W. Q. Wang, and F. L. Bai, Chem. J. Chin. Universities, 1994, 15, 373.

26. T. Yuan, A. M. Weljie, and H. J. Vogel, Biochemistry, 1998, 37, 3187.

27. G. Z. Chen, X. Z. Hang, J. G. Xu, Z. Z. Zheng, and Z. B. Wang, "Method of Fluorescence Analysis", 2nd ed., 1990, Science Press, Beijing. 